\title{
A Rigid Hinge Region is Necessary for High Affinity Binding of Dimannose to Cyanovirin and Associated Constructs
}

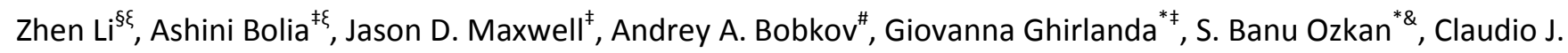
Margulis $^{* \S}$

${ }^{\S}$ Department of Chemistry, University of lowa, lowa City, IA 52242

${ }^{\&}$ Center for Biological Physics, Department of Physics, Arizona State University, Tempe, AZ 85287

${ }^{\ddagger}$ Department of Chemistry and Biochemistry, Arizona State University, Tempe, AZ 85287-1604

"Sanford Burnham Medical Research Institute, 10901 North Torrey Pines Road, La Jolla, CA 92037

Table S1. PCA convergence analysis between the successive slowest modes obtained from the covariance matrices of 100-150ns and 150-200ns windows from the MD simulation.

Table S2. Docking results from BP-Dock using ENM model of the experimental structures and covariance matrix of wt and P51G-m4-CVN from MD simulations.

Figure S1. Average \% $d f i$ values from the last 50 ns of the four simulations for (A) wt-CVN and (B) P51G-m4-CVN. Error bars denote the variation in $\% d f i$ values for the four independent runs.

Figures S2. BP-Dock docking result of the wt-CVN conformation obtained from MD having the crank-shaft motion involving the backbone of Ser52 and Asn53.

Figures S3-S6. CD spectra, thermal denaturation, size exclusion chromatography, SDS-PAGE gel.

Figures S7-S11. ITC titrations with dimannose.

Figure S12. To analyze overall structural variations along MD simulations, we plotted the running average of the RMSD of the backbone fitted to the last frame of our 200ns simulations (each point is an average over $5 \mathrm{~ns}$ ). As is expected from the rigid architecture of CVN, this RMSD is very small throughout each of our independent simulations. (Four colors represent four trials at each condition).

Figure S13. Convergence analysis of distribution in figure 5 of the main text. This same plot is computed at 50, 100, 150 and $200 \mathrm{~ns}$. We see that all features are captured at 50 ns however it is only at times longer than 100 ns that differences between graphs become insignificant for practical analysis. A plot of cumulative differences is also provided. 
Table S1. PCA convergence analysis between the successive slowest modes obtained from the covariance matrices of 100-150ns and 150-200ns windows from the MD simulation.

\begin{tabular}{|c|c|c|}
\hline Protein & Run \# & Correlation \\
\hline P51G-m4-CVN at 300K & $\begin{array}{l}\text { Run } 1 \\
\text { Run } 2 \\
\text { Run } 3 \\
\text { Run } 4\end{array}$ & $\begin{array}{l}0.92 \\
0.94 \\
0.95 \\
0.92\end{array}$ \\
\hline$w t-C V N$ at $300 \mathrm{~K}$ & $\begin{array}{l}\text { Run } 1 \\
\text { Run } 2 \\
\text { Run } 3 \\
\text { Run } 4\end{array}$ & $\begin{array}{l}0.83 \\
0.85 \\
0.81 \\
0.86\end{array}$ \\
\hline P51G-m4-CVN at 330K & $\begin{array}{l}\text { Run } 1 \\
\text { Run } 2 \\
\text { Run } 3 \\
\text { Run } 4\end{array}$ & $\begin{array}{l}0.88 \\
0.86 \\
0.87 \\
0.91\end{array}$ \\
\hline wt-CVN at $330 \mathrm{~K}$ & $\begin{array}{l}\text { Run } 1 \\
\text { Run } 2 \\
\text { Run } 3 \\
\text { Run } 4\end{array}$ & $\begin{array}{l}0.88 \\
0.88 \\
0.83 \\
0.82\end{array}$ \\
\hline
\end{tabular}

Table S2. Docking results from BP-Dock using the Elastic Network Model (ENM) of the experimental structures and covariance matrix of wt and P51G-m4-CVN from MD simulations. Docking score for the covariance matrix docking has been provided as the average value of all the four runs (with standard deviation) for both wt and P51G-m4-CVN. The covariance matrices have been obtained from the last 10ns of each simulation. As the MD simulations are converged, taking the last $50 \mathrm{~ns}$ instead leads to a similar $d f i$ profile. Both BP-Dock using ENM and covariance matrices yield similar results in that wild-type has a more favorable binding energy compared to the mutant.

\begin{tabular}{|c|c|c|}
\hline Protein & $\begin{array}{c}\text { BP-Dock docking } \\
(\mathrm{kcal} / \mathrm{mol})\end{array}$ & $\begin{array}{c}\text { Docking of ensemble generated from Covariance } \\
\text { matrix }(\mathrm{kcal} / \mathrm{mol})\end{array}$ \\
\hline wt-CVN & -291.74 & $-274.02 \pm 6.86$ \\
\hline P51G-m4-CVN & -264.47 & $-234.70 \pm 5.18$ \\
\hline
\end{tabular}



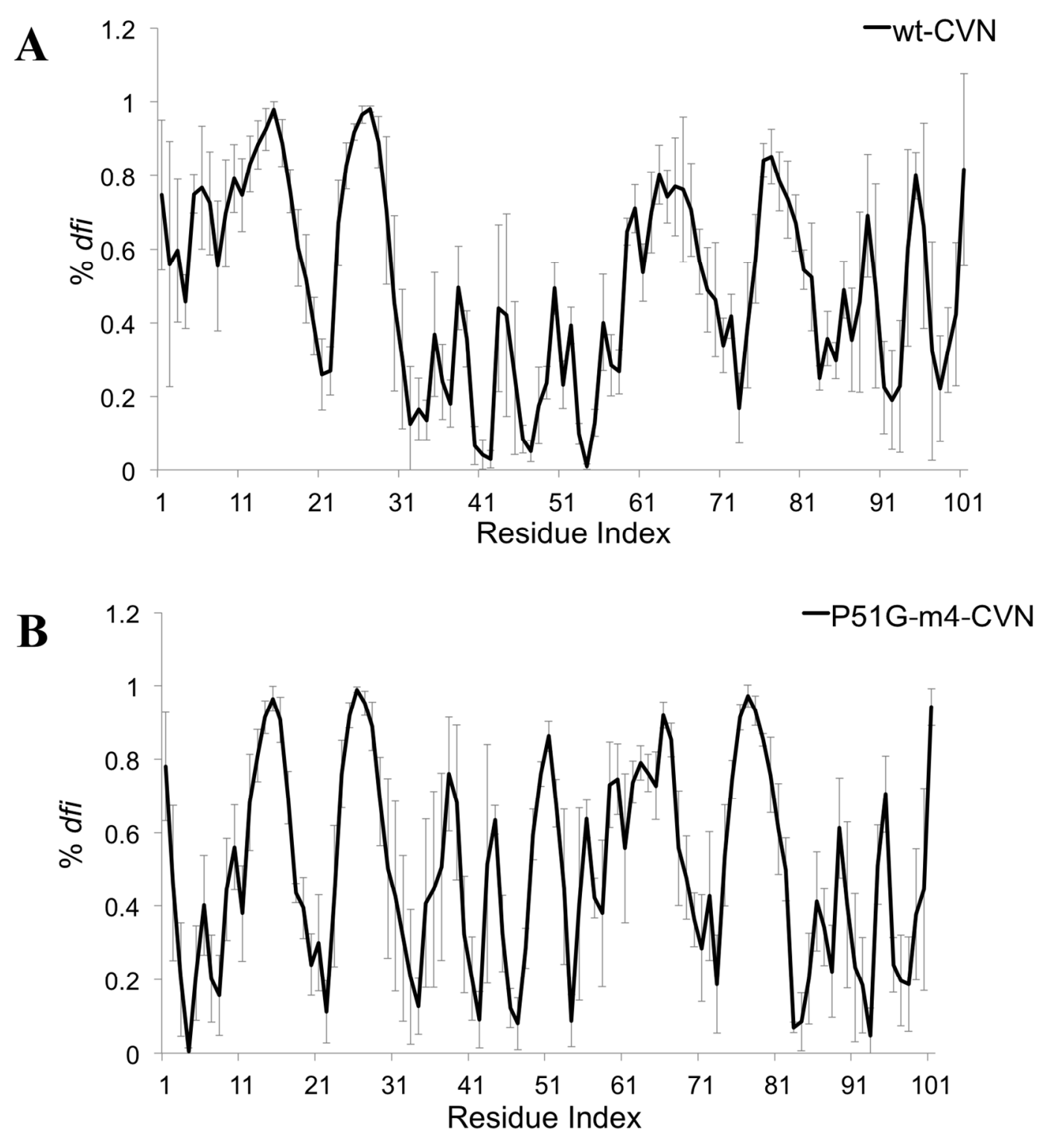

Figure S1. Average \% dfi values from the last 50 ns of the four simulations for (A) wt-CVN and (B) P51G-m4-CVN. Error bars denote the variation in $\% d f i$ values for the four independent runs. Overall as shown in the plot with error bars, the $\% d f i$ profile of wild type is much more rigid around hinge point position 51 compared to that of the mutant. 


\section{NMR structure of wt-CVN}

A

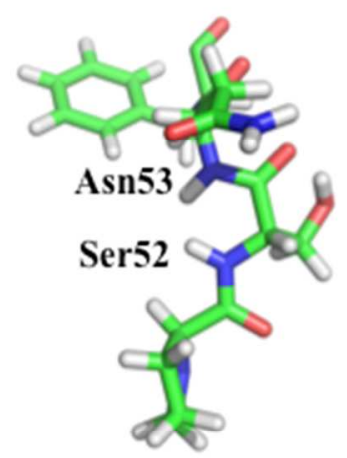

B

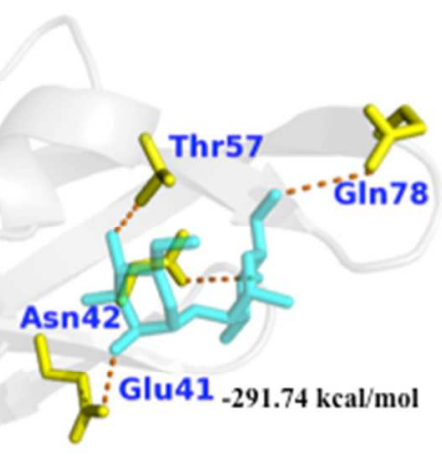

wt-CVN with crank-shaft like motion
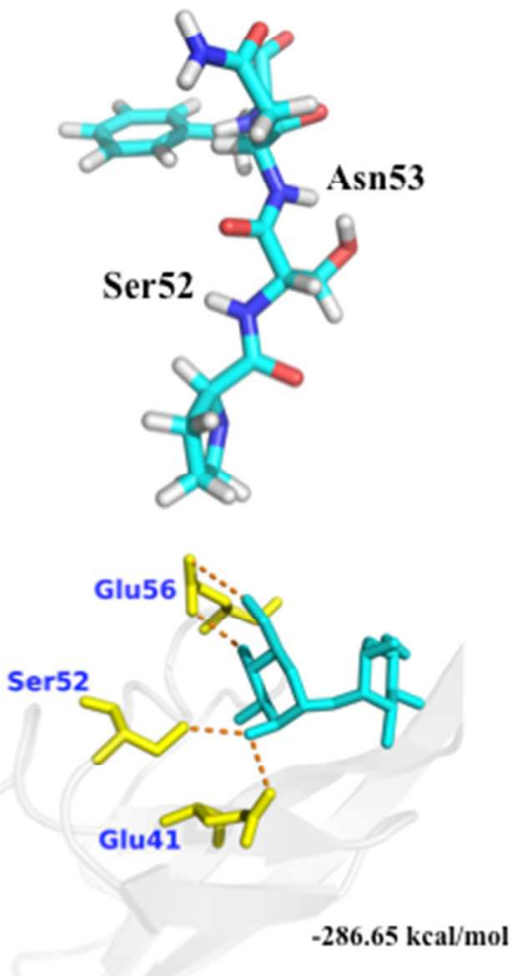

Figure S2. Hinge region of NMR structure of wt-CVN and wt-CVN conformation obtained from MD having the crank-shaft motion involving the backbone of Ser52 and Asn53 (A). The MD conformation relieves the clash between the amide protons of Ser52 and Asn53. Overall, the binding energy of the best-docked pose of the structure of wt-CVN having crank-shaft motion is similar $(-286.65 \mathrm{kcal} / \mathrm{mol})$ to the BP-Dock of the wild type NMR structure $(-291.74 \mathrm{kcal} / \mathrm{mol})(\mathrm{B})$. In both cases, wild type structures (i.e. NMR and amide relieved MD snapshot) yield much more favorable binding energies than that of the mutant. 


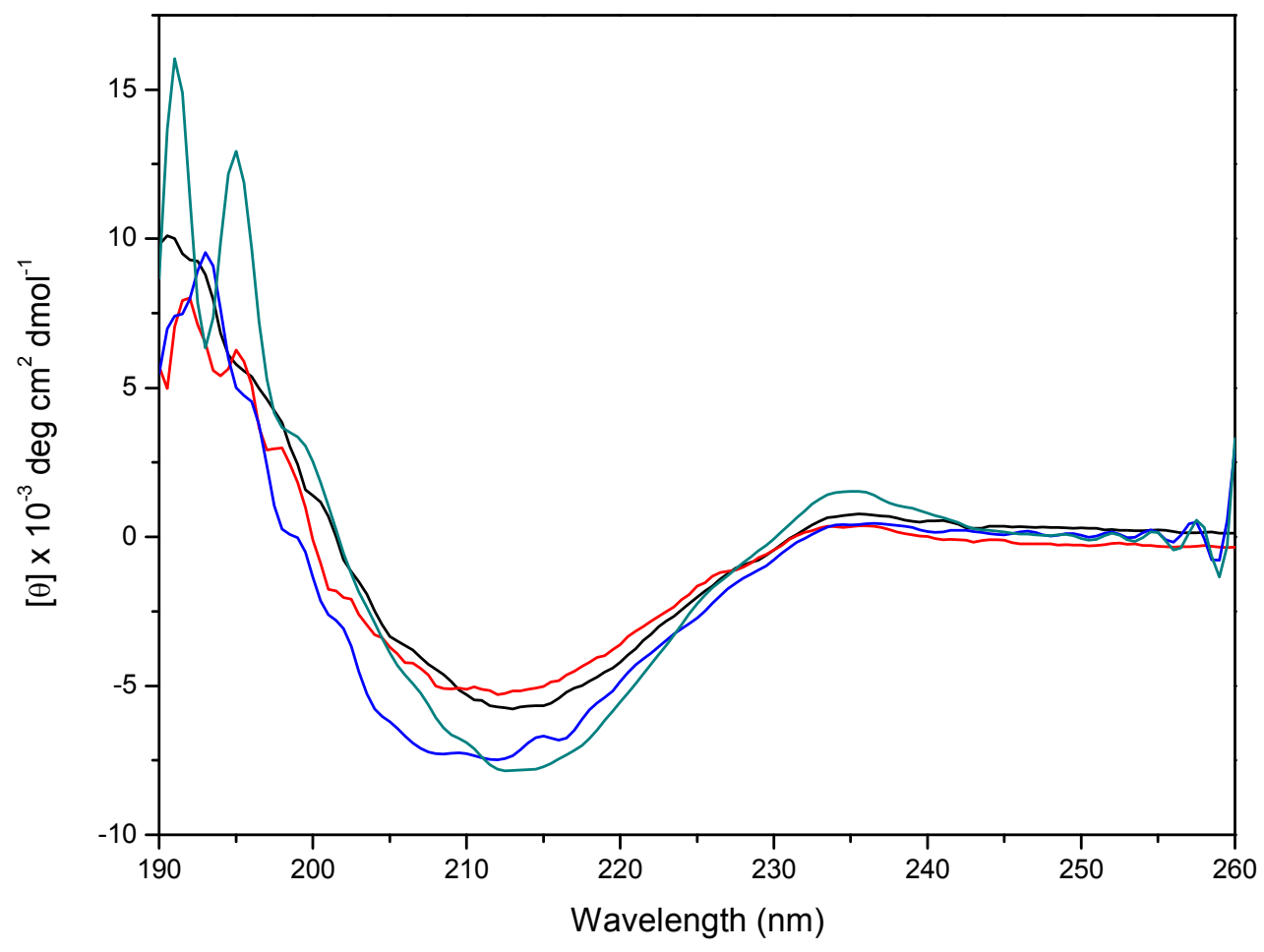

Fig S3. CD of CVN mutants. wt (black), P51Gm4 (red), m4 (blue), P51G (purple) 


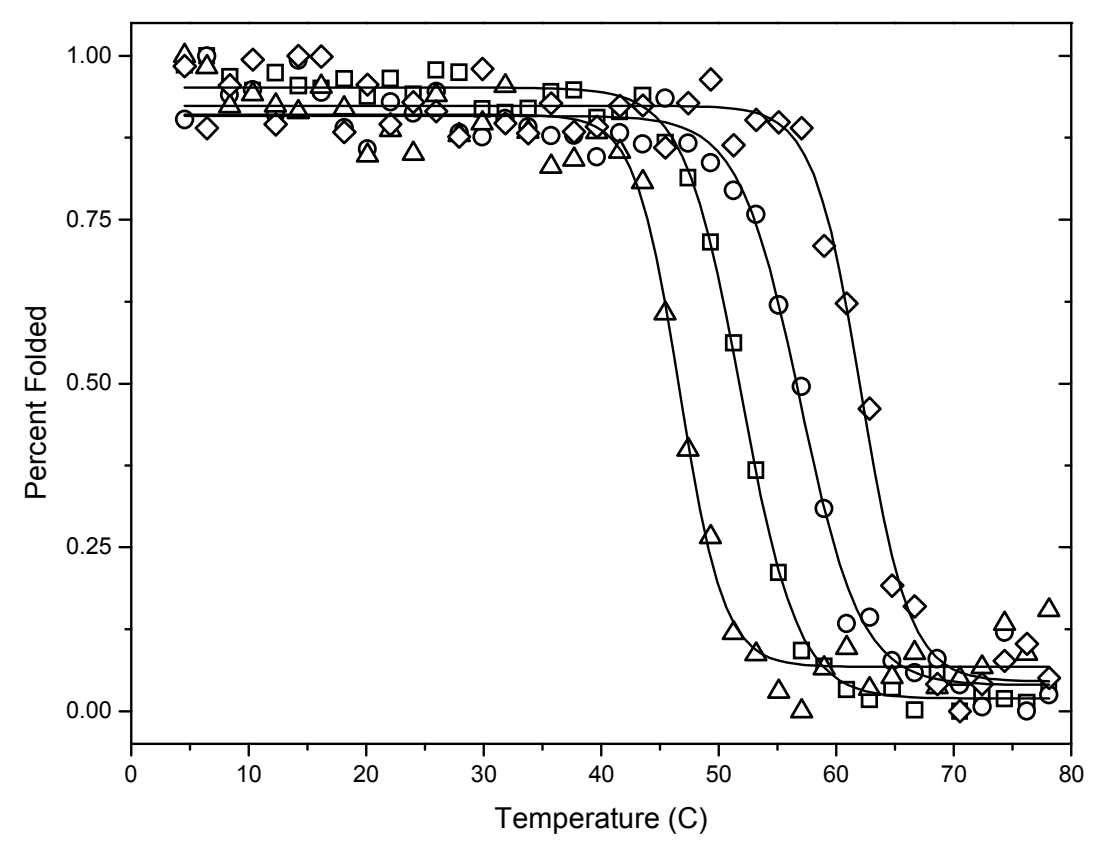

Fig S4. CD Tmelt at CD Signal $202 \mathrm{~nm}$. wt (square), P51Gm4 (circle), m4 (triangle), P51G (diamond) 

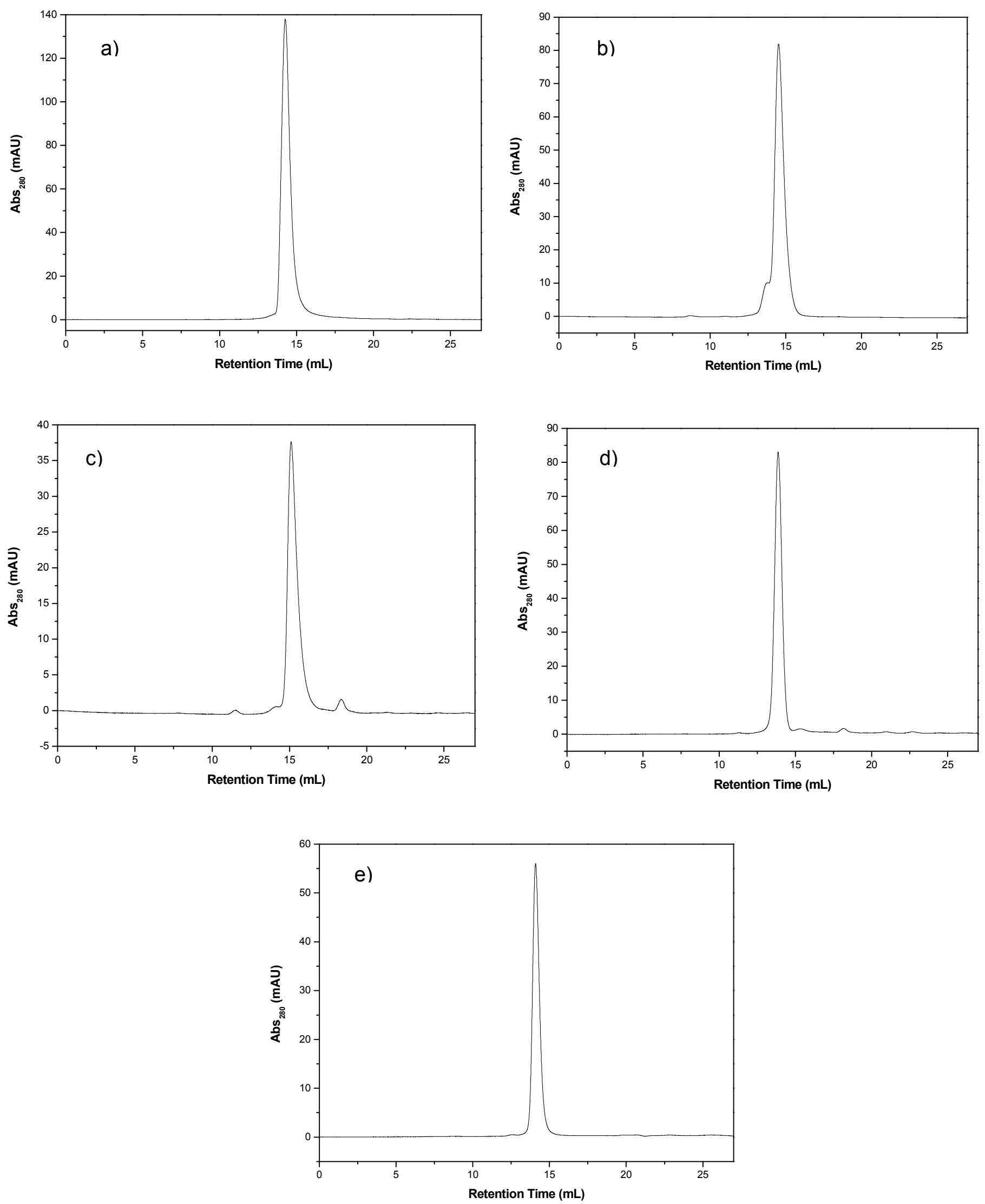

Fig S5. Gel Filtration. a) wt, b) P51Gm4, c) m4 (monomer), d) m4 (dimer), e) P51G 


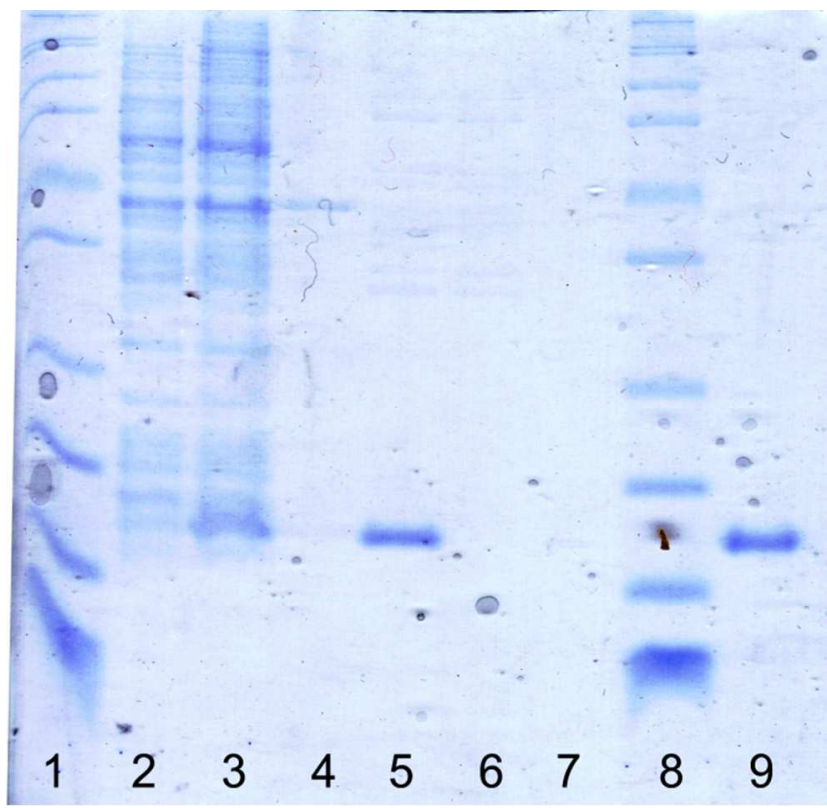

Fig S6. SDS-PAGE of wt Periplasmic Extration. Lanes: 1) Mark12 MW Standard, 2) Pre-IPTG, 3) Post-IPTG, 4) Pellet Wash, 5) Periplasm Extraction, 6,7) IMAC Washes, 8) MW, 9) IMAC Elution 
ITC data and conditions.

ITC titrations were run in duplicate. For simplicity, only one data set is reported. Temperature for all runs was set to 23 ${ }^{\circ} \mathrm{C}$. Data were fit using the Microcal fitting tool, which utilizes Origins. The tool provides two distinct models, "One set of sites with same affinity" and "two sets of sites with different affinities". "One set of sites model" describes binding by one or more binding sites per protein molecule, but they all have same affinity. "Two sets of sites model" is applied when there is one set of sites (one or more sites) with one affinity and a second set of sites (again one or more sites) with affinities higher or lower than in the first set of sides. All data were fit first with "one set of sites with same affinity" model. This model assumes that there are one or more sites per molecule but they have the same affinity. If the fit was good no further analysis was required. If satisfactory fit was not achieved "two sets of sides with different affinity" model was used.

Detailed information on individual titrations are reported below.

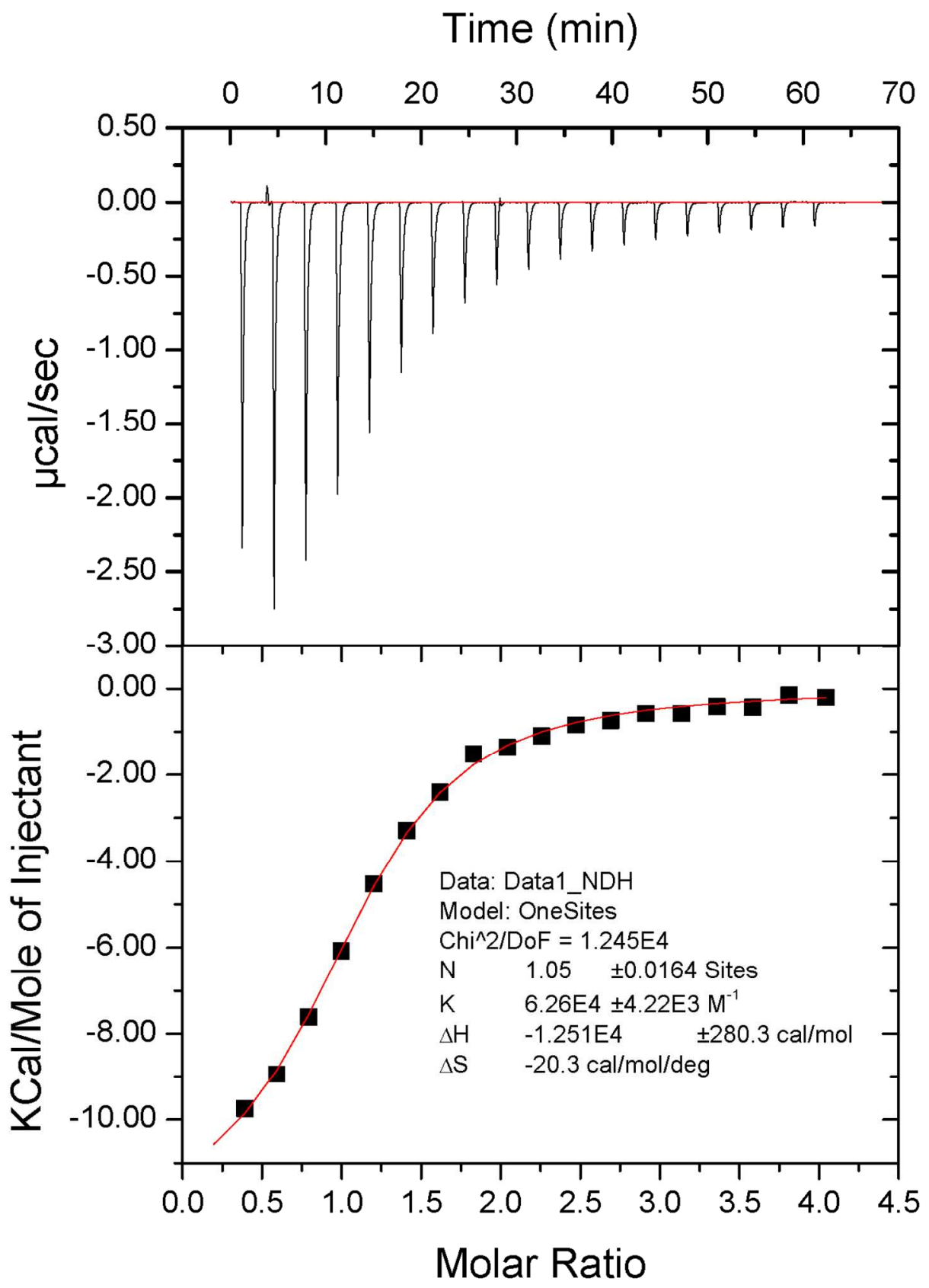


Fig S7. WT CVN with dimannose

Three runs:

1) Injecting $2 \mathrm{mM}$ dimannose to $100 \mathrm{uM}$ wt, 19 injections of $2 \mathrm{ul}$

2) Injecting $20 \mathrm{mM}$ dimannose to $100 \mathrm{uM}$ wt, 19 injections of $2 \mathrm{ul}$

3) Injecting $10 \mathrm{mM}$ dimannose to $100 \mathrm{uM}$ wt, 39 injections of $1 \mathrm{ul}$

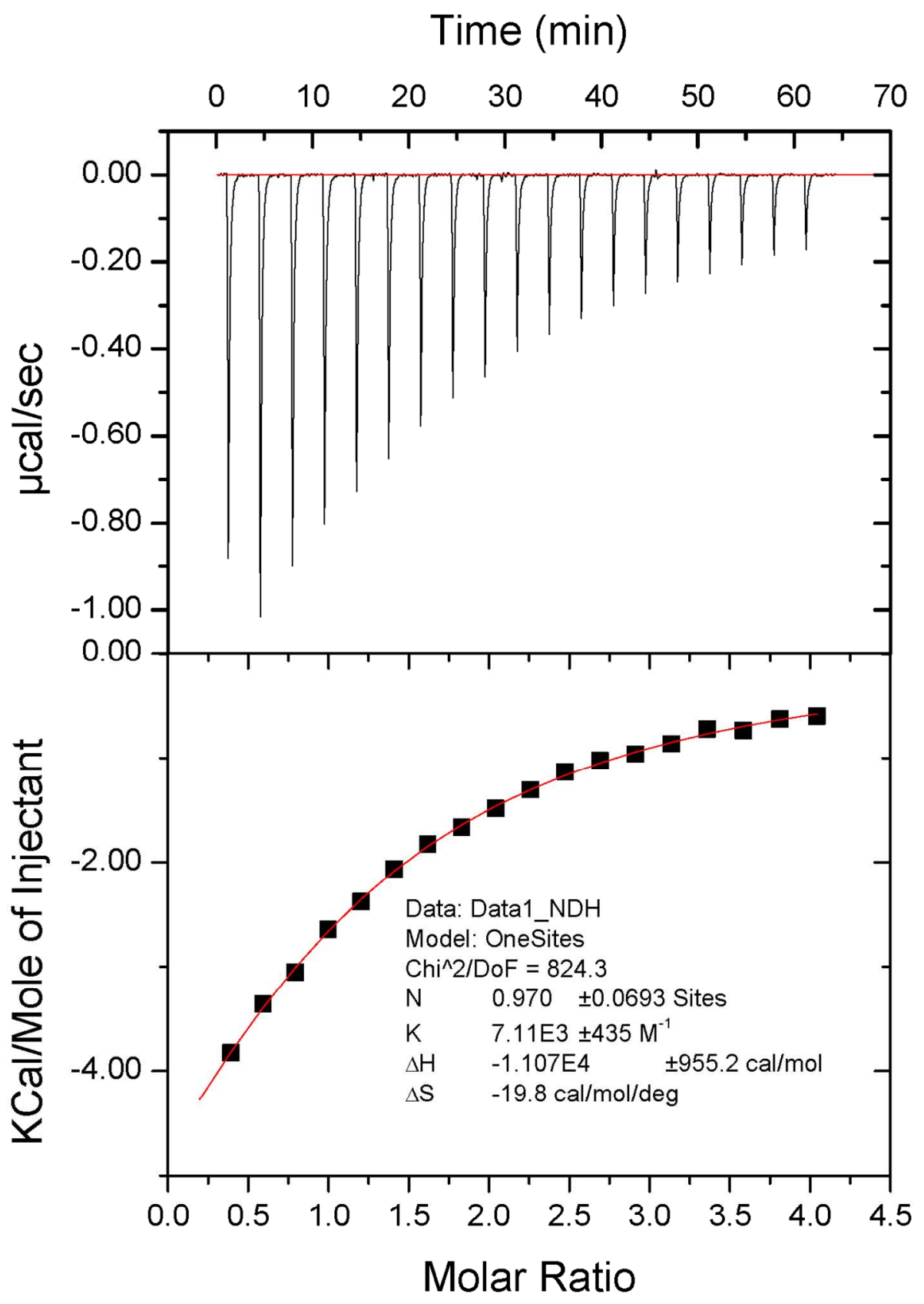

Fig S8. P51Gm4CVN with dimannose

Two runs: 
1) Injecting $3 \mathrm{mM}$ dimannose to $82 \mathrm{uM}$ P51Gm4, 19 injections of $2 \mathrm{ul}$

2) Injecting $10 \mathrm{mM}$ dimannose to $82 \mathrm{uM} \mathrm{P} 51 \mathrm{Gm} 4,39$ injections of $1 \mathrm{ul}$

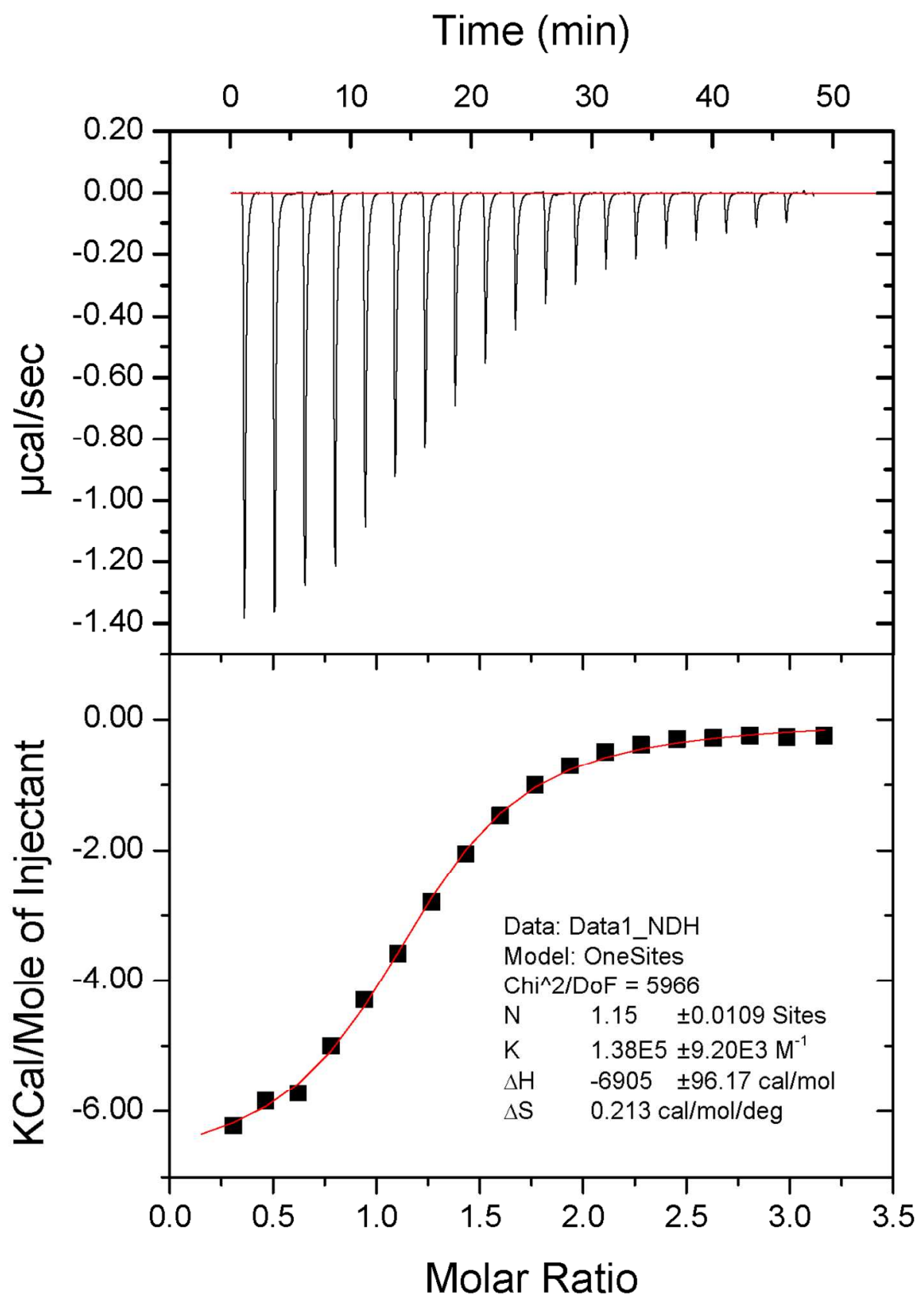

Fig S9. CVN-m4 (monomer) with dimannose

Two runs: 
1) Injecting $1.5 \mathrm{mM}$ dimannose to $98 \mathrm{uM} \mathrm{M} 4,19$ injections of $2 \mathrm{ul}$

2) Injecting $1.0 \mathrm{mM}$ dimannose to $98 \mathrm{uM} \mathrm{M} 4,19$ injections of $2 \mathrm{ul}$

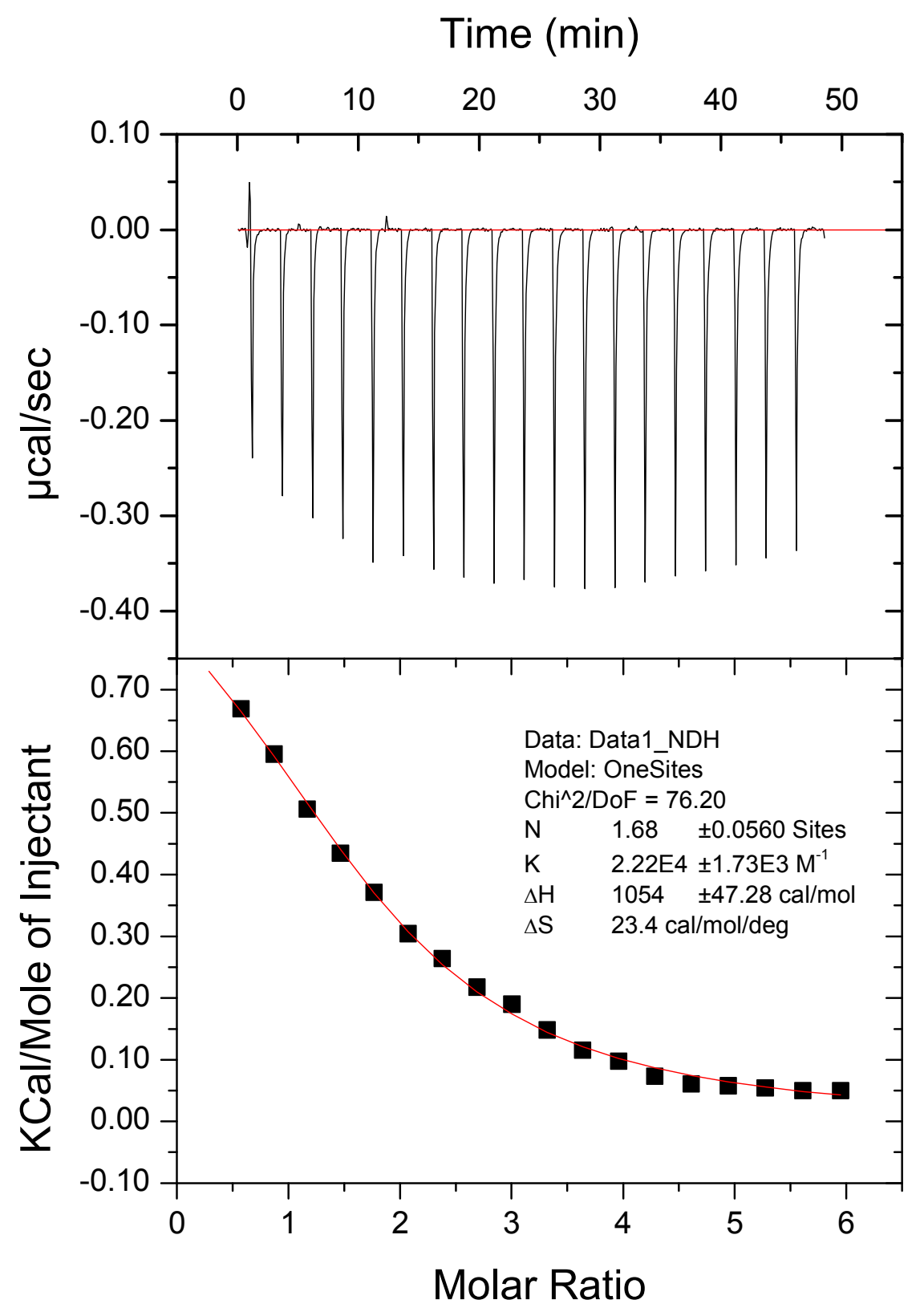


Fig S10. CVN- m4 (dimer) with dimannose

Two runs were made under same conditions: Injecting 2mM dimannose to $69 \mathrm{uM} \mathrm{M} 4,19$ injections of $2 \mathrm{ul}$.

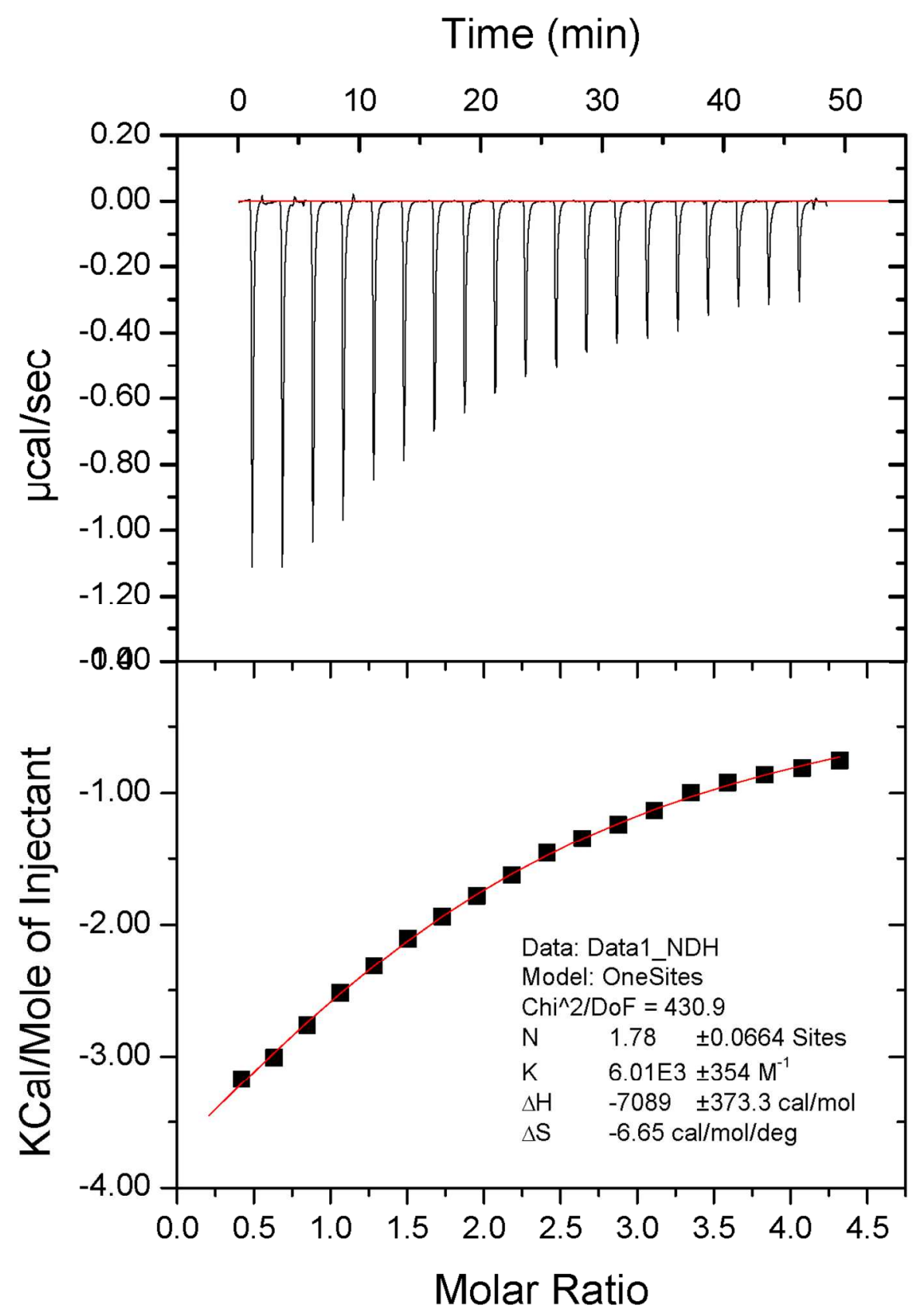

Fig S11. P51G-CVN with dimannose 
Two runs:

1) Injecting $2 \mathrm{mM}$ dimannose to 95uM P51G-CVN, 19 injections of $2 \mathrm{ul}$

2) Injecting $20 \mathrm{mM}$ dimannose to $95 \mathrm{uM}$ P51G-CVN, 19 injections of $2 \mathrm{ul}$
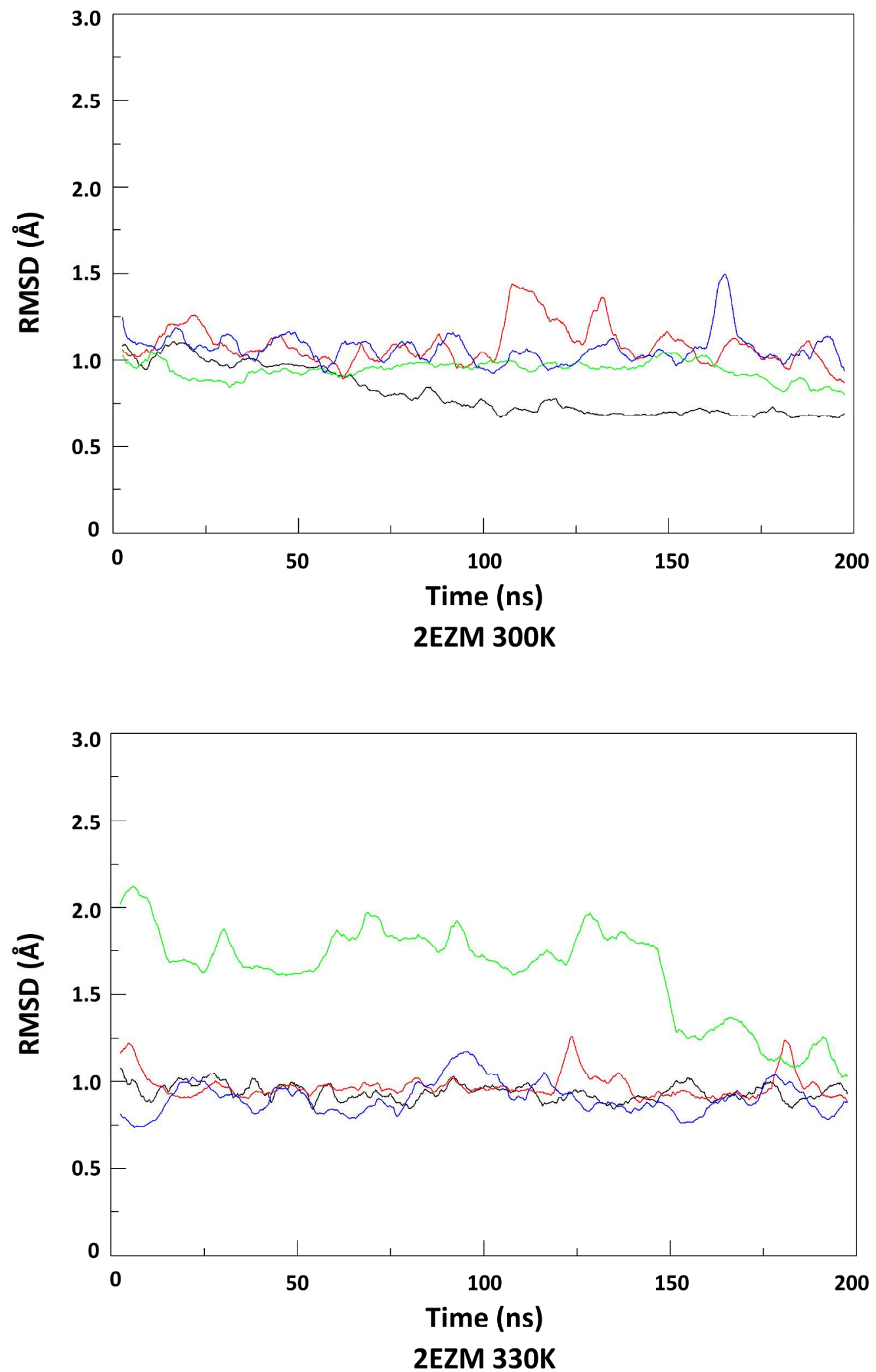


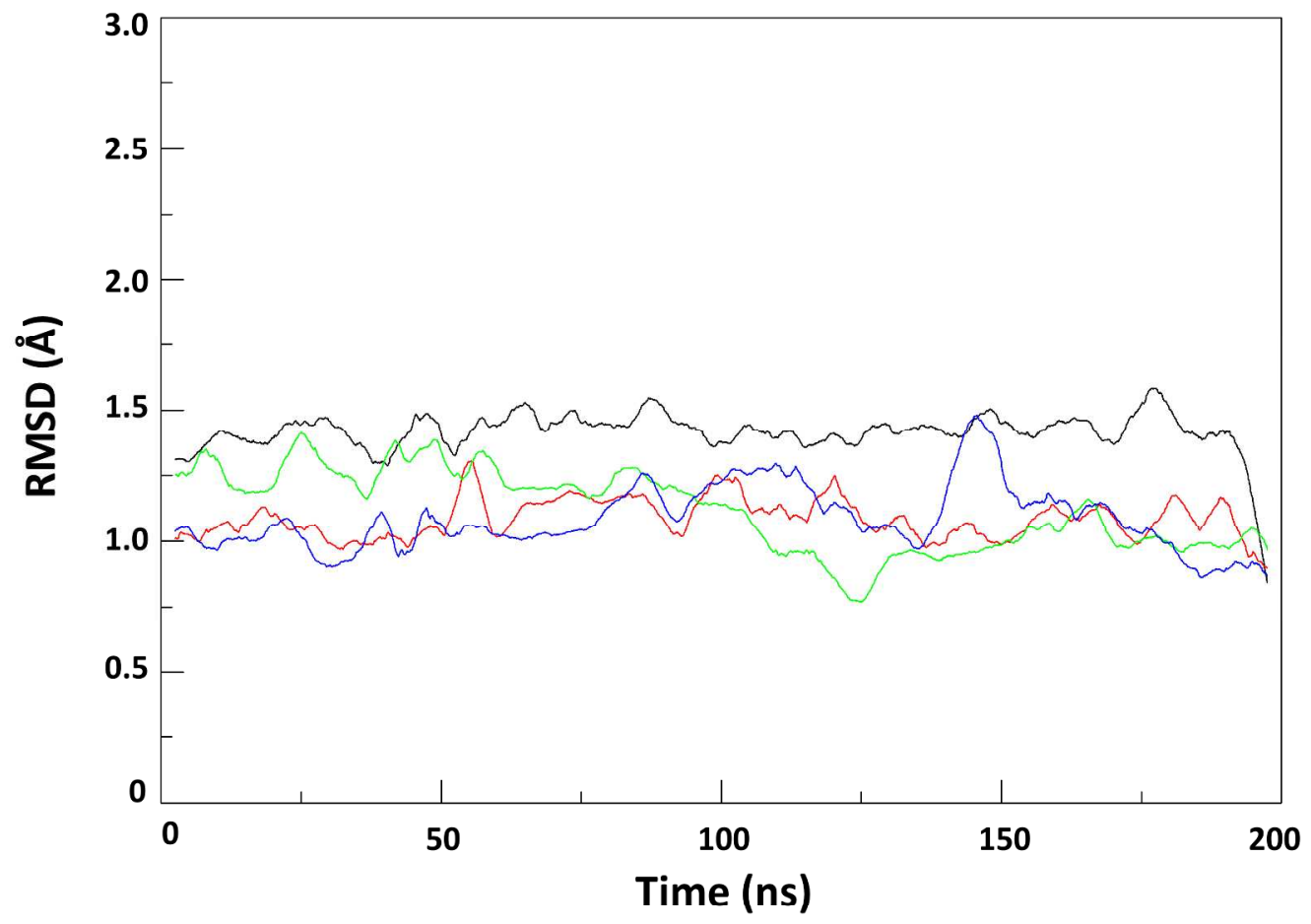

$2 \mathrm{Z21} 300 \mathrm{~K}$

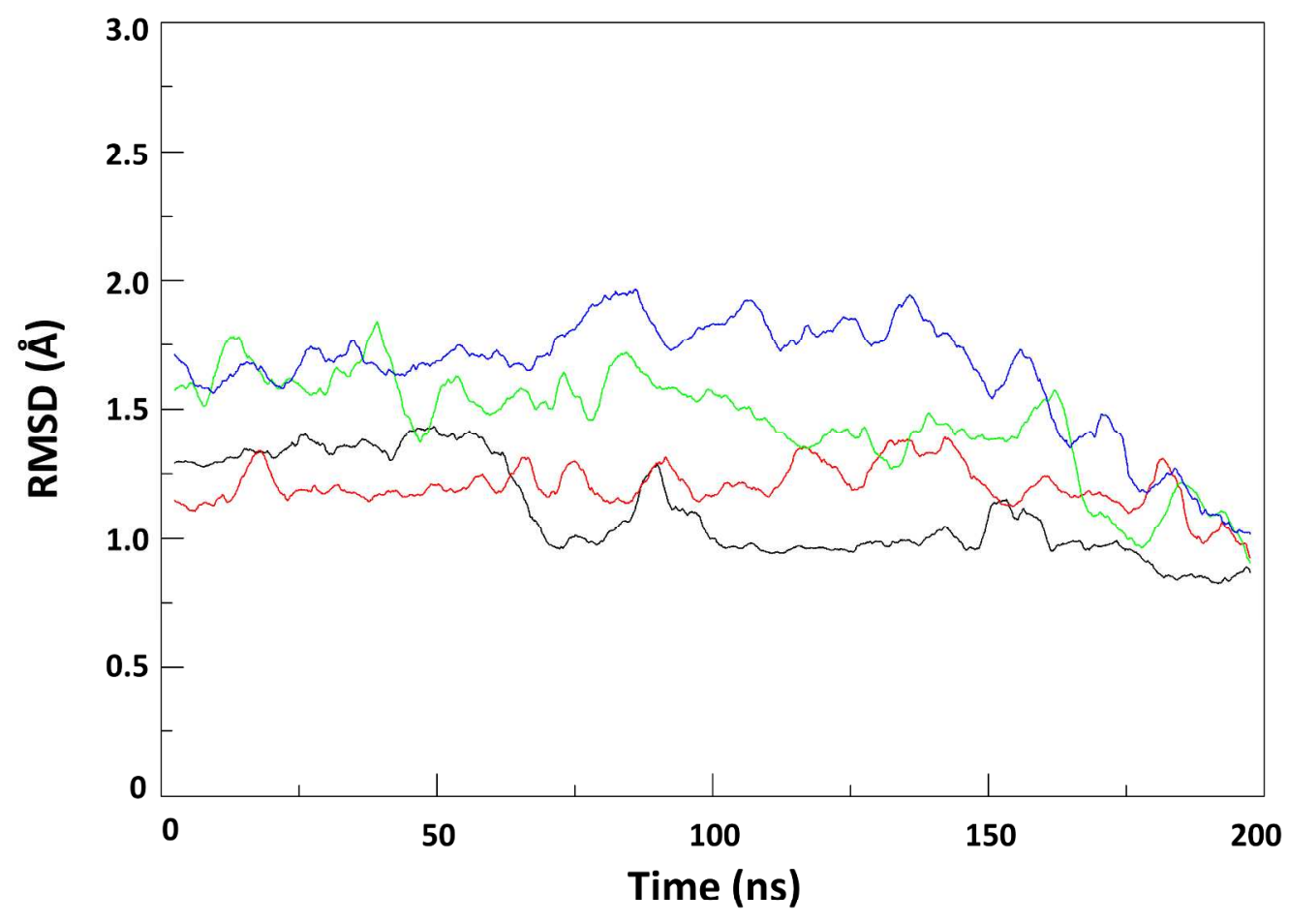

$2 \mathrm{Z21} 330 \mathrm{~K}$

Fig S12. To analyze overall structural variations along MD simulations, we plotted the running average of the RMSD of the backbone fitted to the last frame of our 200ns simulations (each point is an average over $5 \mathrm{~ns}$ ). As is expected from the rigid architecture of CVN, this RMSD is very small throughout each of our independent simulations. (Four colors represent four trials at each condition). 

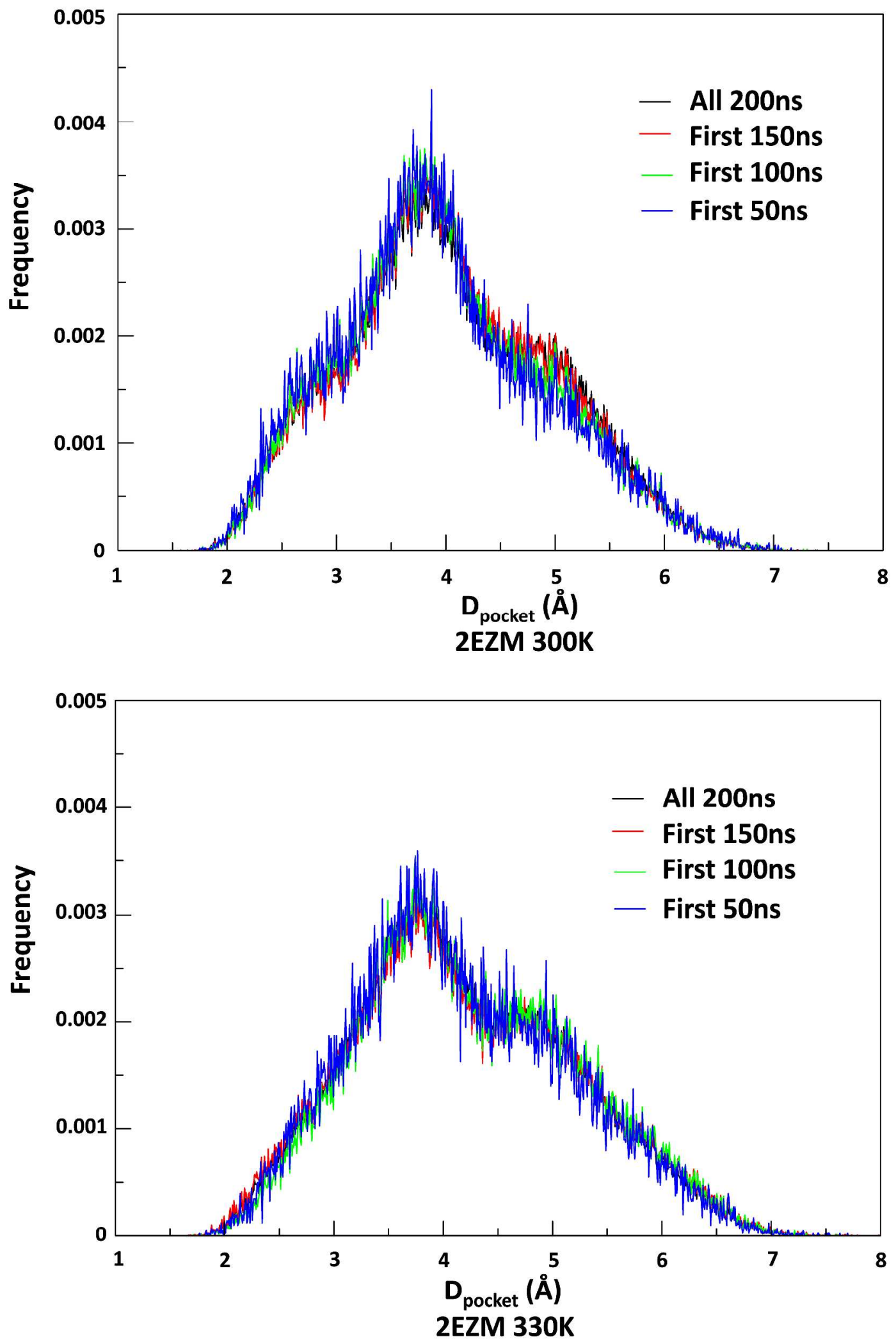

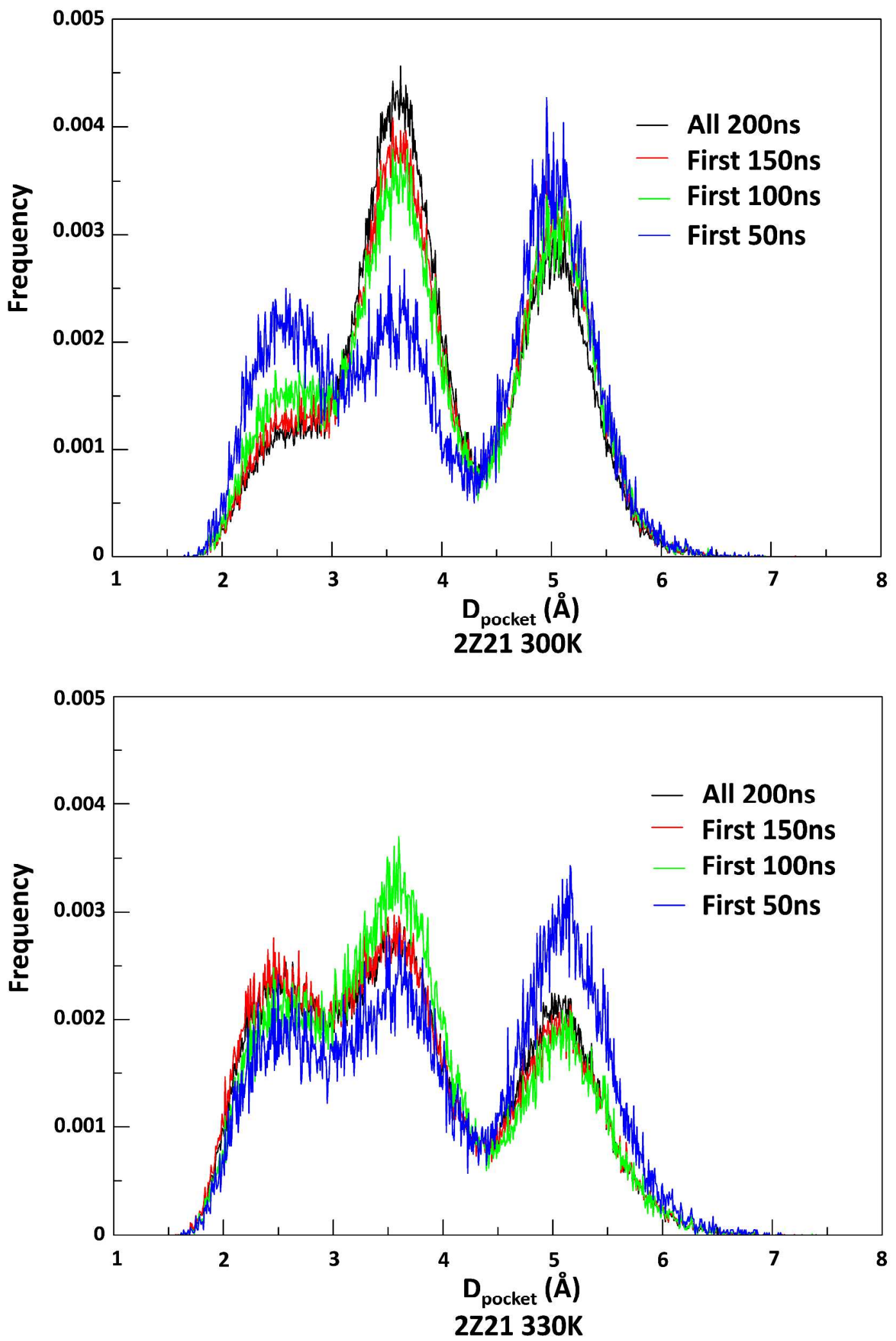


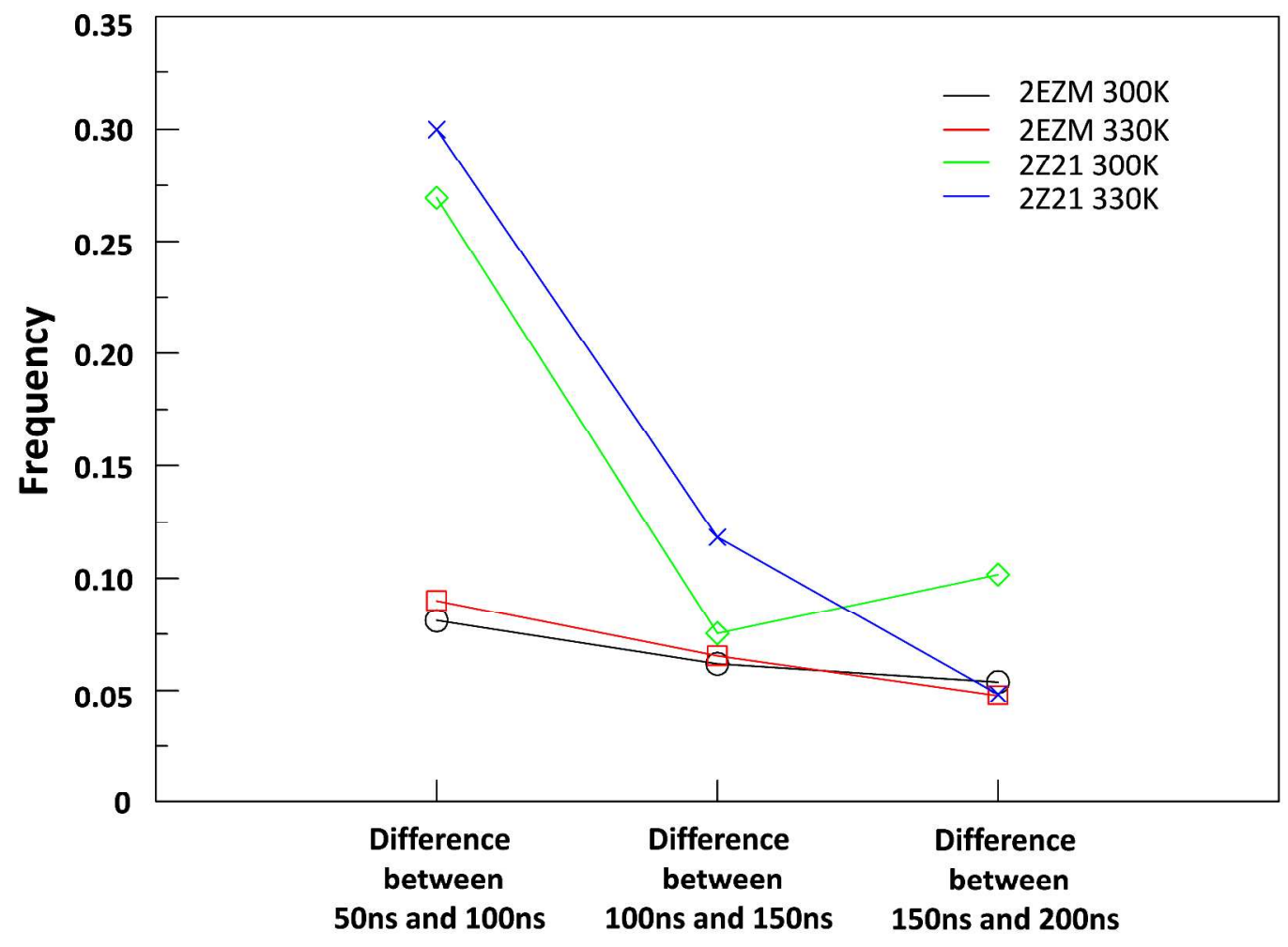

Fig S13. Convergence analysis of distribution in figure 5 of the main text. This same plot is computed at 50, 100, 150 and $200 \mathrm{~ns}$. We see that all features are captured at 50 ns however it is only at times longer than $100 \mathrm{~ns}$ that differences between graphs become insignificant for practical analysis. A plot of cumulative differences is also provided. 